\title{
Worldwide Burden of Gastric Cancer Attributable to Tobacco Smoking in 2012 and Predictions for 2020
}

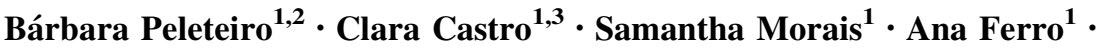 \\ Nuno Lunet ${ }^{1,2}$
}

Received: 18 August 2014/Accepted: 3 March 2015/Published online: 19 March 2015

(C) Springer Science+Business Media New York 2015

\begin{abstract}
Background The heterogeneous patterns and trends in tobacco consumption contribute to regional and gender differences in the burden of gastric cancer attributable to smoking.

Aims To estimate the proportion and absolute number of gastric cancer cases that can be attributed to smoking in different countries, in 2012 and 2020.

Methods Population attributable fractions (PAFs) were computed for 118 countries, using data of smoking prevalence in 2002 and 2011 and published estimates of the
\end{abstract}

Electronic supplementary material The online version of this article (doi:10.1007/s10620-015-3624-x) contains supplementary material, which is available to authorized users.

Bárbara Peleteiro

barbarap@med.up.pt

Clara Castro

clara.castro@ipoporto.min-saude.pt

Samantha Morais

samantha.morais@ispup.up.pt

Ana Ferro

ana.ferro@ispup.up.pt

Nuno Lunet

nlunet@med.up.pt

1 EPIUnit - Institute of Public Health, University of Porto, Rua das Taipas 135, 4050-600 Porto, Portugal

2 Department of Clinical Epidemiology, Predictive Medicine and Public Health, University of Porto Medical School, Alameda Prof. Hernâni Monteiro, 4200-319 Porto, Portugal

3 North Region Cancer Registry (RORENO) - Portuguese Oncology Institute, Rua Dr. António Bernardino de Almeida, 4200-072 Porto, Portugal magnitude of the association between smoking and gastric cancer, assuming a time lag of $\approx 10$ years.

Results For men, the highest PAF estimates in 2012 were observed in Eastern Asia and the lowest in North America, whereas for women the highest were in Western Europe and the lowest in Africa. Very high Human Development Index (HDI) countries presented the lowest median PAF in men (very high vs. high, medium, and low HDI: 17.2 vs. $20.8 \%, p=0.014)$ and the highest median PAF in women (very high vs. high, medium, and low HDI: 4.3 vs. $1.8 \%$, $p<0.001)$. Estimates for 2020 show a decrease in median PAFs, but the estimated absolute number of cases attributable to smoking in the countries analyzed increased for men $(\approx 154,000$ vs. $\approx 160,000)$ and decreased for women $(\approx 6200$ vs. $\approx 5600)$.

Conclusions Smoking accounts for a larger number of gastric cancer cases among men, and gender differences are expected to increase in the next decade, despite the decrease in PAFs. Intensified efforts to control smoking are needed to further reduce the burden of gastric cancer.

Keywords Smoking - Stomach neoplasms - Population attributable fractions $\cdot$ Predictions

\section{Introduction}

Gastric cancer is the fifth most common malignancy and the third leading cause of cancer death worldwide [1], despite the sustained decline of gastric cancer rates over the past several decades $[2,3]$. The apparent potential for an even greater decrease without specific interventions to control its main determinants contributes to the misperception that this is no longer a public health problem, at least in more developed countries. However, in the most 
recent years, the relative declines were smaller in several settings, and a leveling off is already expected in a few countries [4].

Consumption of salt, fruit, and vegetable were the first exposures to be associated with gastric cancer [5, 6], but the evidence currently available shows a lack of consistent results and a weaker effect than initially thought $[7,8]$, whereas Helicobacter pylori infection joined the gastric carcinogenic model more lately $[9,10]$, and the association may be stronger than expected [11]. More recently, gastric cancer has been classified as a tobacco-related cancer [12], and smoking control policies are also expected to influence the burden of this frequent oncologic disease.

Given the heterogeneous patterns and trends in smoking across countries from different world regions and with distinct levels of economic development [13, 14], we aimed to estimate, for a large number of countries worldwide, the proportion and absolute number of gastric cancer cases that can be attributed to smoking in 2012, and the corresponding figures expected for 2020.

\section{Methods}

Population attributable fractions (PAFs), i.e., estimates of the proportion of gastric cancer cases that can be attributed to smoking, were computed for different countries, according to sex. For this, we used country-specific data on smoking prevalence and gastric cancer incidence and published estimates of the magnitude of the association between smoking and gastric cancer. A time lag of approximately 10 years was assumed, based on the stronger associations observed between smoking and cancer or intestinal metaplasia $[15,16]$ than with the preceding gastric lesions, indicative of an effect later in carcinogenesis.

Estimates of PAF and the absolute number of cases attributable to smoking in each country were computed for those with sex-specific data available for both the prevalence of smoking in 2002 and 2011 and gastric cancer incidence in 2012 and projections for 2020.

\section{Association Between Smoking and Gastric Cancer}

We conducted a systematic review of meta-analyses to obtain estimates of the magnitude of association between smoking and gastric cancer by sex. Seventy-two references were retrieved through PubMed search, from inception to July 2014, and backward citation tracking. Six meta-analyses were identified [15, 17-21], and data extraction was accomplished following a protocol defined a priori. A detailed description of the published meta-analyses is provided in Supplemental Table 1 and the results in Fig. 1.
Only two meta-analyses presented the results separately by sex $[15,19]$; however, one of them was restricted to studies evaluating Japanese populations living in Japan [19]. Therefore, we selected the results from a meta-analysis [15] including cohort studies conducted worldwide; to compute PAFs, we used sex-specific estimates of the relative risk (RR) for the comparison of current smokers with never smokers (men: 1.62; women: 1.20).

\section{Prevalence of Smoking}

Smoking prevalence in 2002 [22] and 2011 [23], separately for men and women aged 15 years or older, was retrieved from two World Health Organization (WHO) sources. Data were available for 144 and 166 countries, respectively, for 2002 and 2011.

\section{Calculation of PAFs}

We estimated the proportional reduction in gastric cancer incidence that would arise if exposure to smoking corresponded to a counterfactual distribution scenario, defined as the exposure distribution that would result in the lowest population risk (e.g., the whole population being lifelong non-smokers). We used the method proposed by Levin [24] and computed PAF using the following equation:

$\mathrm{PAF}=\frac{P(\mathrm{RR}-1)}{P(\mathrm{RR}-1)+1}$

where $P$ is the prevalence of smoking, and RR is the relative risk of the association between smoking and gastric cancer.

\section{Gastric Cancer Incidence}

Estimates of the number of new gastric cancer cases in 2012 and predictions for 2020 for men and women were retrieved from GLOBOCAN 2012 [1], for 118 of the countries with available data on the prevalence of smoking. This was used to compute the absolute number of cases attributable to smoking in these countries, for both periods.

\section{Results}

There were wide geographical and gender differences in PAFs in 2012 (Fig. 2 and Supplemental Tables 2 and 3). In all countries, PAFs were higher for men, among whom the lowest median estimates were observed in North America and the highest in Eastern Asia; the absolute number of cases attributable to smoking reached more than 18,000 in Japan and 80,000 in China. For women, the highest median PAFs were found in Western Europe and the lowest in Africa. 


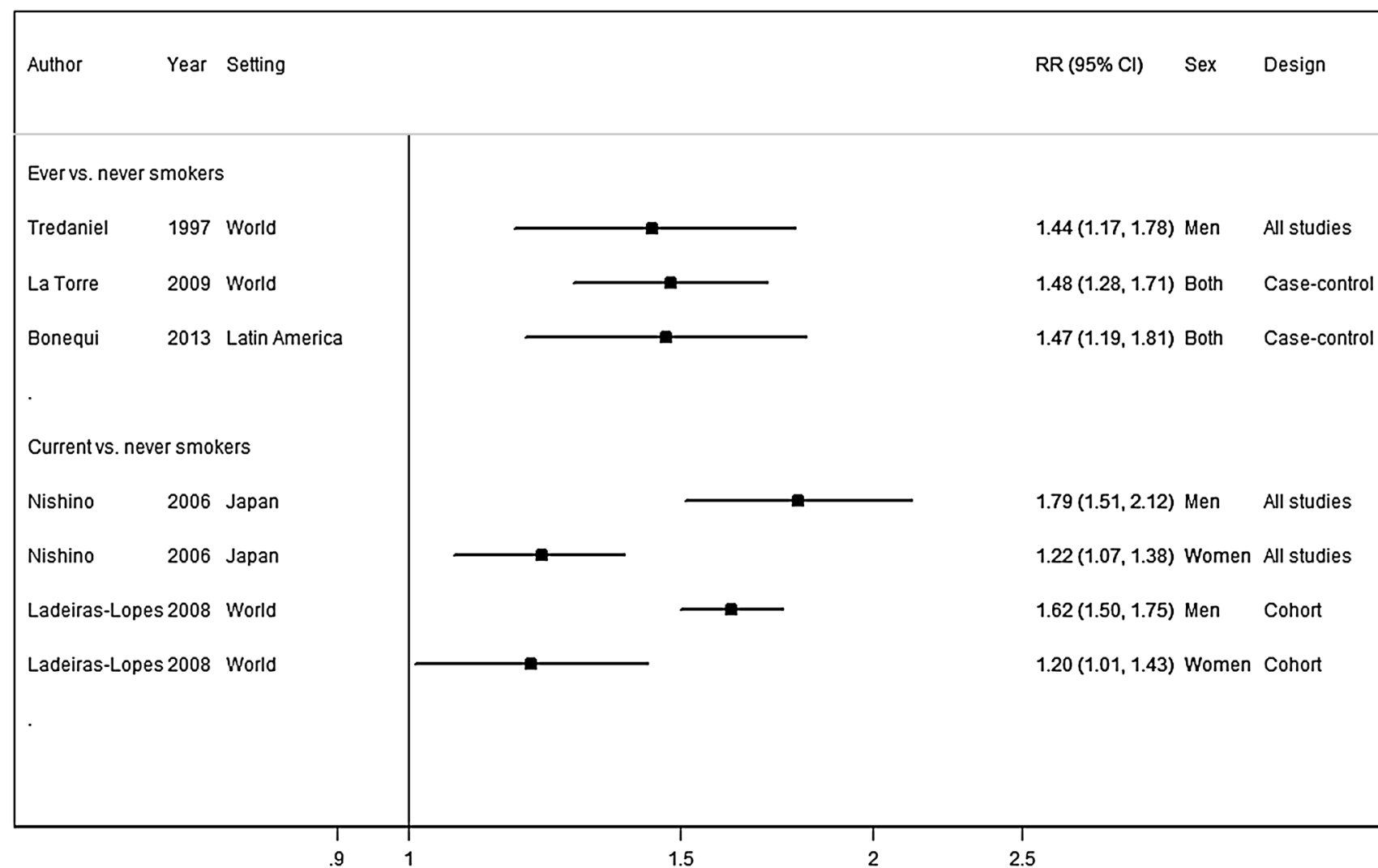

Fig. 1 Meta-analyses [identified through PubMed search, from inception to July 2014, using the following expression: (gastric OR stomach) AND cancer AND (smoking OR tobacco OR cigarette) AND ("systematic review" OR meta-analysis OR "combined analysis" OR "pooled analysis"). Full papers published in English, Portuguese, Spanish, French, Italian, and Polish were evaluated. Screening of

Figure 3 presents PAF estimates according to the levels of Human Development Index (HDI) in 2012 [25]. Countries with high, medium, and low HDI presented similar median PAFs, both among men and women. Very high HDI countries had the lowest median PAF in men (very high vs. high, medium, and low HDI: 17.2 vs. $20.8 \%$, $p=0.014$ ) and the highest median PAF in women (very high vs. high, medium, and low HDI: 4.3 vs. $1.8 \%$, $p<0.001$ ).

Between 2012 and 2020, estimates show a decrease in the median PAF, both in men (from 19.5 to $16.5 \%$ ) and in women (from 3.0 to $1.9 \%$ ); nevertheless, there were several countries, with different levels of HDI, depicting increases in PAFs, except among women from low HDI settings (Fig. 4). In the settings where PAFs were estimated to decrease in this period, the percent variation tended to be more pronounced among women, and the absolute number of cases increased in only a few of these countries. In men, the proportional declines in PAFs were usually smaller than $25 \%$ and the estimated number of cases increased in a greater number of these countries (Fig. 4). reference lists and data extraction were accomplished independently by two researchers (BP and CC), following a protocol defined a priori, and discrepancies were discussed until consensus or resolved involving a third researcher (NL)] on the association between smoking and gastric cancer

In the 118 countries analyzed, the absolute number of gastric cancer cases attributable to smoking increased from $\approx 154,000$ in 2012 to $\approx 160,000$ in 2020 among men and decreased from $\approx 6200$ in 2012 to $\approx 5600$ in 2020 among women. The most pronounced changes were a decrease in $\approx 4000$ cases in Japan and an increase in $\approx 8000$ cases in China, among men.

\section{Discussion}

The proportion of gastric cancer cases that can be attributed to smoking differs substantially between men and women and across countries with different levels of development, as expected when taking into account the gender and regional differences in the prevalence of smoking.

Previous analyses have shown variation according to sex, with $13-16 \%$ of gastric cancer cases being attributed to smoking in men and 4-7\% in women $[21,26]$. Direct comparisons with our results are difficult due to differences in the periods and regions covered by different studies on 


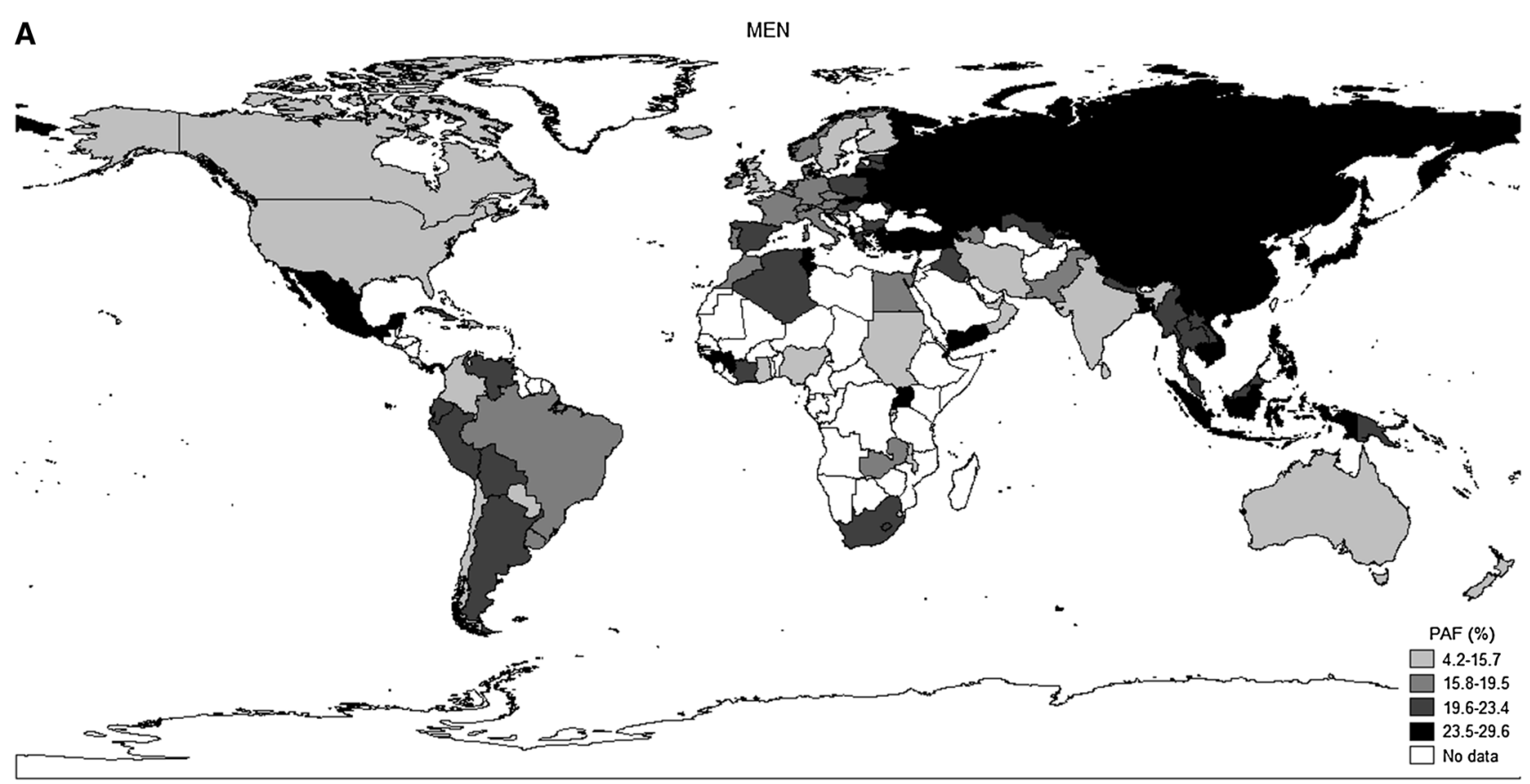

B

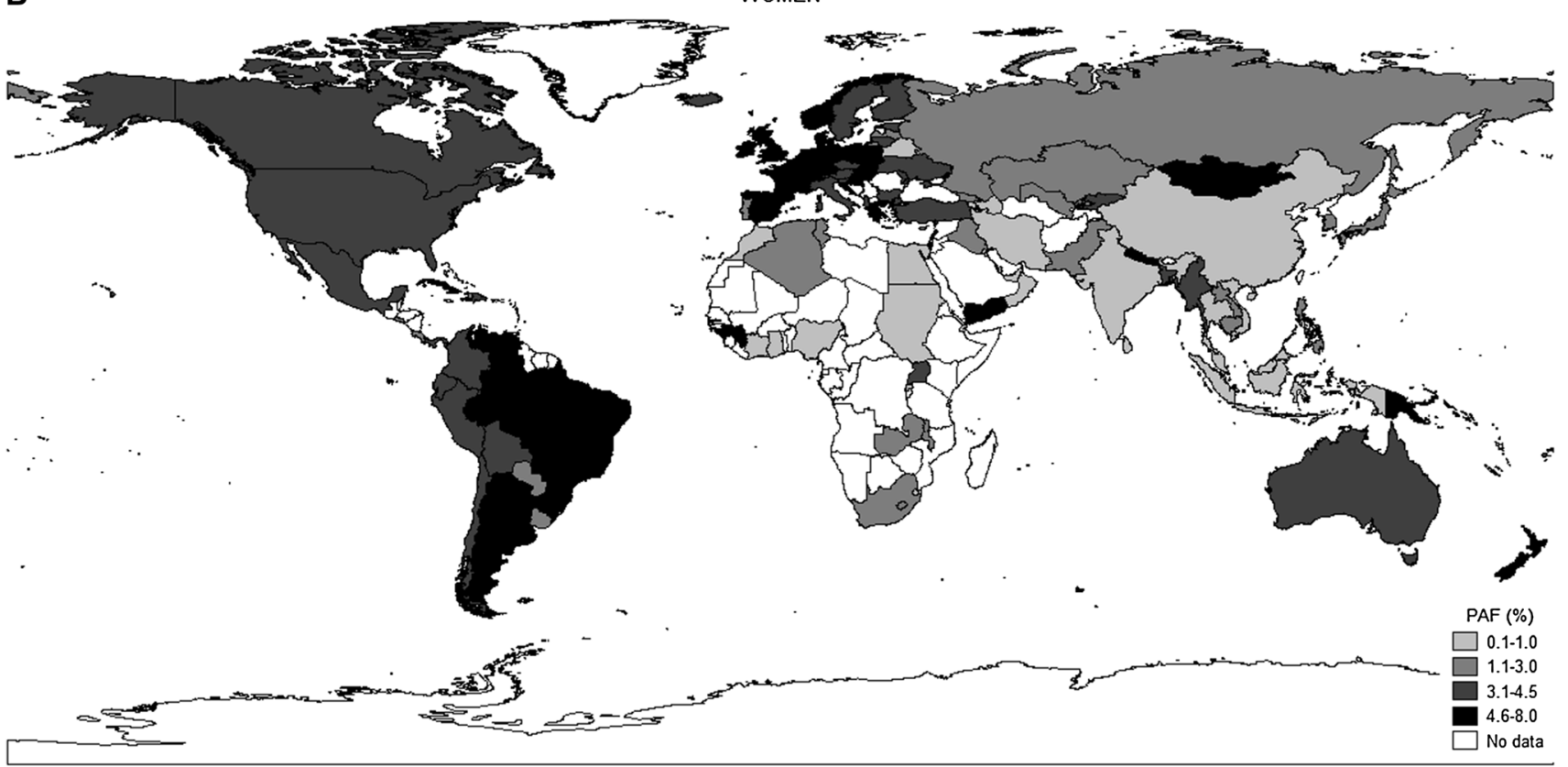

Fig. 2 Estimates of PAFs (quartiles of the distributions among men and women were used as cutoffs) in 118 countries (countries with no PAF estimates are presented in white), in 2012, among men and women

this topic. The burden of gastric cancer attributable to smoking has also been shown to differ with socioeconomic status at country level, being higher in more developed countries compared with those less developed (14 vs. $9 \%$ [21], 25 vs. $11 \%$ [27], or 23 vs. $9 \%$ [26]). Our results confirm previous observations and depict more pronounced differences in women.
Joint analyses of smoking and other risk factors, namely H. pylori infection, contributing to gastric cancer burden have only been conducted in countries where data on the prevalence of these two risk factors are widely available, namely the USA [28]. Such an approach, however, is highly dependent on the availability of a large amount of data on trends in the exposure to the risk factors. Therefore, 

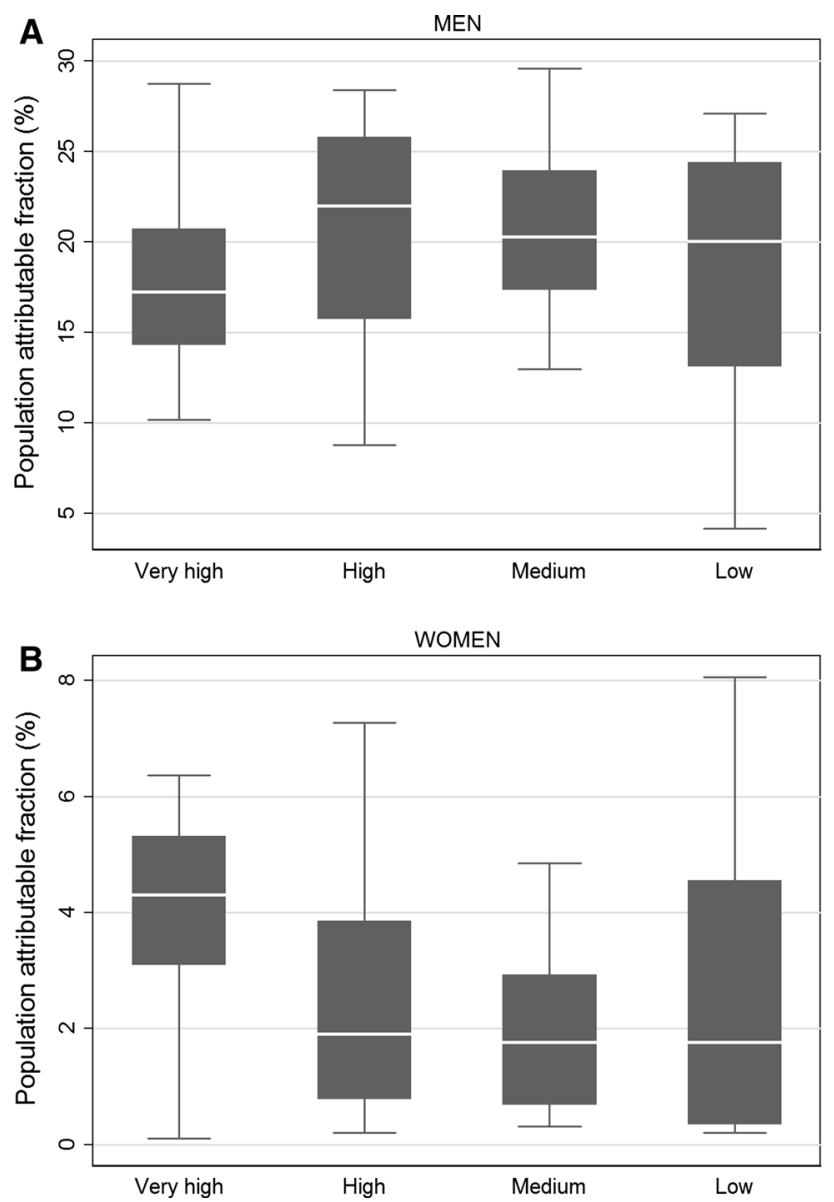

Fig. 3 Estimates of PAFs according to HDI (HDI distribution in 2012 was retrieved from the Human Development Report, 2013 [25]: $42,33,25$, and 18 countries with very high, high, medium, and low HDI, respectively), in 2012, among men and women

computing joint PAFs for $H$. pylori infection and smoking would limit our analyses to a much smaller number of countries, most of them in the very high HDI level, precluding the identification of geographical areas where efforts to reduce gastric cancer burden through the control of smoking should be enhanced.

Downward trends in the prevalence of smoking have been observed worldwide [29], which explain the predicted decrease in PAFs from 2012 to 2020 in most settings. Nevertheless, the variation in the estimates was heterogeneous, with increases in PAFs being estimated for a relatively large number of countries. The absolute number of cases may be expected to increase in countries with higher PAF estimates in 2020 as well as in those with more modest declines in PAFs after 2012; among the latter, the magnitude of the decline in PAFs may be insufficient to overcome the increase in the number of cases due to population growth and aging.

The assumptions underlying valid estimation of PAFs include a causal relationship between exposure and disease,
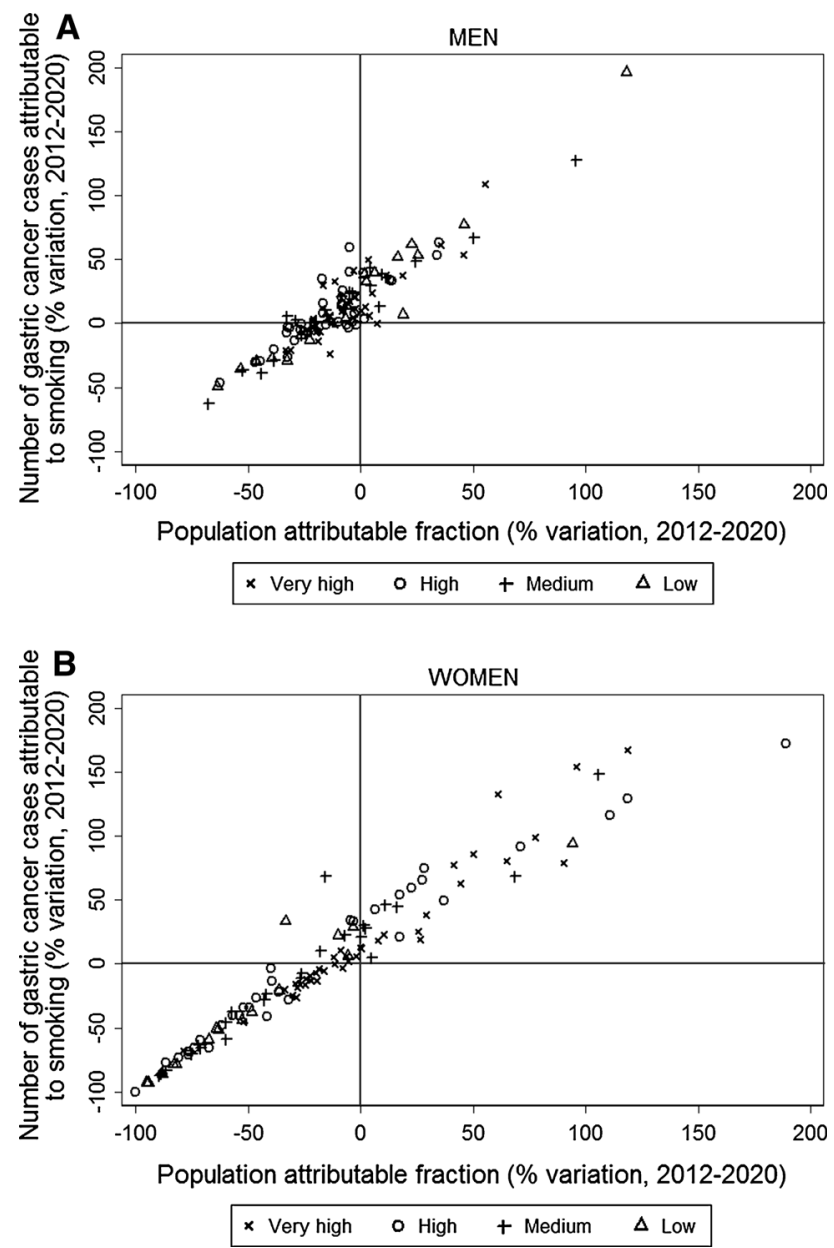

Fig. 4 Relation between the variation in the estimated PAFs and in the number gastric cancer cases attributable to smoking, between 2012 and 2020, according to HDI (HDI distribution in 2012 was retrieved from the Human Development Report, 2013 [25]: 42, 33, 25 , and 18 countries with very high, high, medium, and low HDI, respectively), among men and women

and that unbiased and free from confounding RR estimates are available, for the relation between the exposure and the outcome. Regarding the causal role of smoking in gastric carcinogenesis, in 2004, the International Agency for Research on Cancer classified tobacco smoking as carcinogenic to humans, concluding that there was sufficient evidence that it causes gastric cancer [12]. This evaluation was based on cohort, case-control, and cross-sectional studies conducted in various parts of the world showing a consistent association between smoking and gastric cancer and also presented data regarding the intensity and duration of smoking, smoking cessation, and the effect on gastric cancer precursor lesions. Although residual confounding cannot be ruled out, it is unlikely that control for the effects of potentially major confounders, other than $H$. pylori infection and dietary factors, has not been accomplished. For the present study, we used the best available evidence on 
the association between smoking and gastric cancer, obtained from the most recent meta-analysis of cohort studies [15].

Regarding the validity of the RR estimates for the association between smoking and gastric cancer, these were retrieved from a meta-analysis of cohort studies, for which lower summary estimates were obtained (1.53 [15] for both sexes) compared with other study designs, such as casecontrol studies (1.69 [18] for both sexes). In addition, the summary estimates from the meta-analysis were based on a mixture of crude and adjusted RRs, with summary estimates being higher when based solely on adjusted RRs [15]. Taken together, these may have contributed to conservative PAF estimates, though not compromising the comparison across regions. To further improve the accuracy of our estimates of PAF, we used sex-specific summary RRs, to account for differences in the patterns of smoking between men and women, and we assumed a time lag of approximately 10 years between exposure to smoking and occurrence of cancer, which is supported by the available evidence on the relation between smoking and gastric cancer precursor lesions [16]. This may have contributed to more accurate estimates of the burden of gastric cancer attributable to smoking than in previous studies, which took into account a smaller [21] or no lag time [26].

Lauren's [30] classification system has been widely used in epidemiological research, subdividing tumors into two main histological types-diffuse and intestinal. Although originally a relatively greater impact of environmental factors in the etiology of intestinal-type carcinomas was hypothesized [31], while the diffuse type was considered more dependent on the individuals' genetic profile [32], the currently available evidence shows no substantial etiological differences between these tumors. Despite few studies addressing the effect of environmental exposures on the risk of specific gastric cancer histological types, small differences in the magnitude of the association between smoking and gastric cancer according to histological type have been observed, generally with the intestinal type showing higher risk estimates [33-35]. However, when finer markers of intestinal differentiation, such as CDX2 expression, are used, no statistically significant or consistent associations between environmental exposures and CDX2 expression status are observed, suggesting that gastric cancer subtypes are essentially homogeneous regarding the relation with most of the established determinants [36]. Furthermore, the lack of worldwide gastric cancer incidence estimates according to histological type precludes the quantification of the effect of smoking in gastric cancer subtypes at the international level.

Systematic reviews have also shown no meaningful differences in the magnitude of the association between current smoking and cardia and non-cardia gastric cancer
$[15,18]$; nevertheless, point estimates for the RR are higher for cardia cancers compared with non-cardia. This may have contributed to the country-specific PAFs computed in this study to be underestimated in countries where a higher proportion of cardia cancers is observed (e.g., Australia, Finland, USA, UK) and overestimated in countries where cardia cancers represent a small proportion of all gastric cancers (e.g., Japan, Russia) [4].

The accuracy of PAF estimates is also dependent on the quality of the data obtained from each source, and the use of current smoking prevalence has some limitations. Although smoking prevalence was obtained mainly from national surveys conducted within each country, different methodologies may have been adopted across studies, namely regarding age group restriction and definition of current smoking. Furthermore, the accumulated hazards of smoking for cancers, which have relatively long latency, depend on the age at which smoking began, duration of smoking, number of cigarettes smoked per day, and type of tobacco product used (cigarettes, cigars, bidis, etc.). These limitations may have contributed to inaccuracies in the individual estimates, but are unlikely to compromise the definition of general patterns, particularly with respect to differences across genders, geographical regions, levels of HDI, and between 2012 and 2020.

Gastric cancer is currently interpreted as a multifactorial complex disease, and therefore, different sets of causal mechanisms leading to its occurrence may coexist [11]. Under this assumption, the same exposure (e.g., smoking) may contribute to the incidence of gastric cancer when occurring together with different sets of other exposures (e.g., H. pylori infection or $H$. pylori infection and fruit consumption or $H$. pylori infection and salt intake). Though the different causal mechanisms potentially leading to gastric cancer are not known, the estimated PAFs reflect a scenario where smoking is not present, therefore affecting all of the possible pathways to cancer, regardless of the persistence by the remaining exposures that take part in those causal mechanisms. Therefore, our results provide information on the importance of smoking as a cause of gastric cancer, and the comparison between 2012 and 2020 estimates shows how improvements in tobacco control strategies may gradually contribute to reduce the burden of gastric cancer. However, our estimates do not correspond to realistically attainable objectives through public health interventions, since a scenario with a $0 \%$ prevalence of smoking is unlikely.

In conclusion, smoking accounts for a much larger number of gastric cancer cases among men, and this number is expected to increase in the next decade, despite the decrease in PAFs. Intensified efforts to control smoking are needed to further reduce the burden of gastric cancer. 
Acknowledgments This work was supported by "Fundação para a Ciência e a Tecnologia" (PTDC/SAU-EPI/122460/2010 and SFRH/ BPD/75918/2011).

Conflict of interest None.

\section{References}

1. International Agency for Research on Cancer. GLOBOCAN 2012 v1.0, Cancer Incidence and Mortality Worldwide: IARC CancerBase No. 11, 2012. http://globocan.iarc.fr. Accessed January 10, 2014.

2. Bertuccio P, Chatenoud L, Levi F, et al. Recent patterns in gastric cancer: a global overview. Int J Cancer. 2009;125:666-673.

3. Peleteiro B, Severo M, La Vecchia C, Lunet N. Model-based patterns in stomach cancer mortality worldwide. Eur J Cancer Prev. 2014;23:524-531.

4. Ferro A, Peleteiro B, Malvezzi M, et al. Worldwide trends in gastric cancer mortality (1980-2011), with predictions to 2015, and incidence by subtype. Eur J Cancer. 2014;50:1330-1344.

5. Howson CP, Hiyama T, Wynder EL. The decline in gastric cancer: epidemiology of an unplanned triumph. Epidemiol Rev. 1986;8:1-27.

6. Correa P, Haenszel W, Cuello C, Tannenbaum S, Archer M. A model for gastric cancer epidemiology. Lancet. 1975;2:58-60.

7. Lunet N, Lacerda-Vieira A, Barros H. Fruit and vegetables consumption and gastric cancer: a systematic review and metaanalysis of cohort studies. Nutr Cancer. 2005;53:1-10.

8. Lunet N, Valbuena C, Vieira AL, et al. Fruit and vegetable consumption and gastric cancer by location and histological type: case-control and meta-analysis. Eur J Cancer Prev. 2007;16: 312-327.

9. Correa P. Human gastric carcinogenesis: a multistep and multifactorial process-First American Cancer Society Award Lecture on Cancer Epidemiology and Prevention. Cancer Res. 1992;52: 6735-6740.

10. IARC Working Group on the Evaluation of Carcinogenic Risks to Humans. Schistosomes, liver flukes and Helicobacter pylori. IARC Monogr Eval Carcinog Risks Hum. 1994;61:1-241.

11. Peleteiro B, La Vecchia C, Lunet N. The role of Helicobacter pylori infection in the web of gastric cancer causation. Eur $J$ Cancer Prev. 2012;21:118-125.

12. IARC Working Group on the Evaluation of Carcinogenic Risks to Humans. Tobacco smoke and involuntary smoking. IARC Monogr Eval Carcinog Risks Hum. 2004;83:1-1438.

13. Lopez AD, Collishaw NE, Piha T. A descriptive model of the cigarette epidemic in developed countries. Tob Control. 1994;3: 242-247.

14. Thun M, Peto R, Boreham J, Lopez AD. Stages of the cigarette epidemic on entering its second century. Tob Control. 2012;21: 96-101.

15. Ladeiras-Lopes R, Pereira AK, Nogueira A, et al. Smoking and gastric cancer: systematic review and meta-analysis of cohort studies. Cancer Causes Control. 2008;19:689-701.

16. Morais S, Rodrigues S, Amorim L, Peleteiro B, Lunet N. Tobacco smoking and intestinal metaplasia: systematic review and meta-analysis. Dig Liver Dis. 2014;46:1031-1037.

17. Bonequi P, Meneses-Gonzalez F, Correa P, Rabkin CS, Camargo MC. Risk factors for gastric cancer in Latin America: a metaanalysis. Cancer Causes Control. 2013;24:217-231.
18. La Torre G, Chiaradia G, Gianfagna F, et al. Smoking status and gastric cancer risk: an updated meta-analysis of case-control studies published in the past ten years. Tumori. 2009;95:13-22.

19. Nishino Y, Inoue M, Tsuji I, et al. Tobacco smoking and gastric cancer risk: an evaluation based on a systematic review of epidemiologic evidence among the Japanese population. Jpn J Clin Oncol. 2006;36:800-807.

20. Tramacere I, La Vecchia C, Negri E. Tobacco smoking and esophageal and gastric cardia adenocarcinoma: a meta-analysis. Epidemiology. 2011;22:344-349.

21. Tredaniel J, Boffetta P, Buiatti E, Saracci R, Hirsch A. Tobacco smoking and gastric cancer: review and meta-analysis. Int $J$ Cancer. 1997;72:565-573.

22. Mackay J, Eriksen M. The tobacco atlas. Geneva: World Health Organization; 2002.

23. WHO report on the global tobacco epidemic. Enforcing bans on tobacco advertising, promotion and sponsorship. Geneva: World Health Organization; 2013.

24. Levin ML. The occurrence of lung cancer in man. Acta Unio Int Contra Cancrum. 1953;9:531-541.

25. United Nations Development Programme. Human Development Report 2013, 2013. http://hdr.undp.org/en/statistics/. Accessed January 23, 2014.

26. Ott JJ, Ullrich A, Mascarenhas M, Stevens GA. Global cancer incidence and mortality caused by behavior and infection. $J$ Public Health (Oxf). 2011;33:223-233.

27. Danaei G, Vander Hoorn S, Lopez AD, Murray CJ, Ezzati M, Group CRAC. Causes of cancer in the world: comparative risk assessment of nine behavioural and environmental risk factors. Lancet. 2005;366:1784-1793.

28. Yeh JM, Hur C, Schrag D, et al. Contribution of H. pylori and smoking trends to US incidence of intestinal-type noncardia gastric adenocarcinoma: a microsimulation model. PLoS Med. 2013;10:e1001451.

29. $\mathrm{Ng} \mathrm{M}$, Freeman MK, Fleming TD, et al. Smoking prevalence and cigarette consumption in 187 countries, 1980-2012. JAMA. 2014; 311:183-192.

30. Lauren P. The two histological main types of gastric carcinoma: diffuse and so-called intestinal-type carcinoma. An attempt at a histo-clinical classification. Acta Pathol Microbiol Scand. 1965;64:31-49.

31. Correa P, Sasano N, Stemmermann GN, Haenszel W. Pathology of gastric carcinoma in Japanese populations: comparisons between Miyagi prefecture, Japan, and Hawaii. J Natl Cancer Inst. 1973;51:1449-1459.

32. Tahara E. Genetic pathways of two types of gastric cancer. IARC Sci Publ. 2004;157:327-349.

33. Inoue M, Tajima K, Yamamura Y, et al. Influence of habitual smoking on gastric cancer by histologic subtype. Int J Cancer. 1999;81:39-43.

34. Koizumi Y, Tsubono Y, Nakaya N, et al. Cigarette smoking and the risk of gastric cancer: a pooled analysis of two prospective studies in Japan. Int J Cancer. 2004;112:1049-1055.

35. Ye W, Ekstrom AM, Hansson LE, Bergstrom R, Nyren O. Tobacco, alcohol and the risk of gastric cancer by sub-site and histologic type. Int J Cancer. 1999;83:223-229.

36. Peleteiro B, Lunet N, Wen X, et al. Association between environmental factors and CDX2 expression in gastric cancer patients. Eur J Cancer Prev. 2012;21:423-431. 